\title{
A statistical view on the galactic cold ISM distribution
}

\author{
L. Viktor Tóth, Sarolta Zahorecz, Gábor Marton and Erika Verebélyi \\ Department of Astronomy, Eötvös University, Budapest \\ email: 1.v.toth@astro.elte.hu
}

\begin{abstract}
Inhomogenities were found in the distribution of the cold fraction in the interstellar medium (traced by Planck cold clumps). In large scales there is a significant overdensity on some shells, in small scales there are elongated groups. Critical column density for star formation is $N\left(\mathrm{H}_{2}\right)=7 \times 10^{21} \mathrm{~cm}^{-2}$.
\end{abstract}

Keywords. ISM: dust, ISM: molecules, ISM: bubbles, ISM: clouds, stars: formation

Recent Planck (Planck Collaboration 2011a) and Herschel (Pilbratt et al. 2010) measurements uncovered the distribution of cold interstellar medium (CISM) in the Galaxy by its FIR and sub-mm radiation. We investigated the all sky distribution of cold clumps (ECC and C3PO, Planck Collaboration 2011b) in all scales using statistical methods, and compared it to the distribution of known galactic structures and that of the young stellar objects (YSOs). Comparing the cold clump distribution to the GMCs as traced by $\mathrm{CO}$ we find that besides the overall correlations there are regions with peculiarly low or high CISM content. Large shells and voids traced by HI $21 \mathrm{~cm}$ radiation (see e.g. Heiles 1984) and $100 \mu \mathrm{m}$ surface brightness (Könyves et al. 2007) are often associated with CISM. About $1 / 5$ of the shells are bearing cold clumps, and some of those are very active sites in the formation of dense CISM. Cold clump clusters were located and investigated using the minimum spanning tree method as described in Gutermuth et al. (2009). The distribution of ECC clumps is dominated by elongated groups in the Taurus region. The CISM distribution is well correlated with the distribution of YSOs selected by their WISE (Wright et al. 2010) and AKARI FIS (Yamamura et al. 2010) colours (Tóth et al. 2012). All the ECC clumps that were surveyed by Wu et al. (2012) were checked for associated star formation, and a critical column density for star formation of $N\left(\mathrm{H}_{2}\right)=7 \times 10^{21} \mathrm{~cm}^{-2}$ was found, i.e. all clumps over that column density have associated young stellar objects. This research was supported by the grants HAS-JSPS118, OTKA K101393 and TAMOP-4.2.1/B-09/1/KMR of the EU and the European Social Fund.

\section{References}

Gutermuth, R. A., Megeath, S. T., Muzerolle, J., et al. 2009, ApJS 184, 18

Heiles, C. 1984, ApJS 55, 585

Könyves, V., Kiss, Cs., Moór, A., et al. 2007, A\&A 463, 1227

Pilbratt, G. L., Riedinger, J. R., Passvogel, T., et al. 2010, A\&A 518, L1

Planck Collaboration 2011a, A\&A 536, 1

Planck Collaboration 2011b, A $\& A$ 536, 23

Tóth, L. V., Zahorecz, S., Marton, G., et al. 2012, in prep.

Wright, E. L., Eisenhardt, P. R. M., Mainzer, A. K., et al. 2010, AJ 140, 1868

Wu, Y., Liu, T., Meng, F., et al. 2012, ApJ 756, 76

Yamamura., et al. 2010, AKARI/FIS All-Sky Survey Point Source Catalogues (ISAS/JAXA) 